\title{
Todo fallo es político. Fragmentos de ecología política en la Suprema Corte de Justicia de Mendoza
}

Every judicial sentence is political. Fragments of Political Ecology from Mendoza's Supreme Court

Mariano Javier Salomone INCIHUSA, CONICET,

CCT-Mendoza

msalomone@mendoza-conicet.gob.ar

\section{Resumen}

En 2015 la Suprema Corte de Justicia de Mendoza ratificó la constitucionalidad de la Ley 7722, que prohíbe el uso de sustancias tóxicas en la explotación de minería metalífera. En esta provincia el conflicto social alrededor de proyectos de minería en gran escala adquiere desde 2003 una especial relevancia histórica. El artículo propone un análisis de la hegemonía discursiva que construye el Estado a través de uno de sus brazos, el poder judicial. Para ello realiza una lectura crítica del fallo identificando las referencias principales que tejen su argumentación, organizan su sentido y lo vinculan a cierta ecología política, en tanto trama ideológica que sostiene y organiza una determinada racionalidad en la apropiación social de la naturaleza.

Palabras clave: megaminería - Ley 7722 - juridificación - conflicto social Mendoza

Fecha de recepción:

5.4.18

\section{Abstract}

In 2015 the Mendoza Province Supreme Court of Justice confirmed the constitutionality of Act 7722, which bans the use of toxic substances in metalliferous mining

14.11.18 
activities. The social conflict raised since 2003 by large scale mining projects in Mendoza gives this ruling a special historical significance. This paper analyzes the discoursive hegemony built by the state through the judicial branch, by means of a critical interpretation of the Court's ruling, identifying the main references that support its legal arguments, organize its sense, and connect it to certain political ecology in so far it is an ideological fabric that gives support and organizes some rationality with regards to the social appropriation of Nature.

Key words: mega-mining; Act 7722 - juridification social conflict - Mendoza

\section{Introducción}

En diciembre de 2015, la Suprema Corte de Justicia de Mendoza (en adelante SCJM) ratificó la legalidad de la Ley provincial 7722, al rechazar la demanda de inconstitucionalidad que interpuso una decena de empresas mineras que veían frenados o afectados sus respectivos proyectos de explotación metalífera, dada la prohibición de uso de sustancias tóxicas que aquella norma establece. Así, el Máximo Tribunal provincial intervenía en dirección opuesta a la política que asumía el recientemente electo gobierno nacional del presidente Mauricio Macri, quien en febrero de 2016, mediante el Decreto 349/2016, anunció la eliminación de las retenciones a la minería. Mientras la intervención del poder judicial local ratificaba las condiciones al avance de la megaminería en la provincia, el ejecutivo nacional iniciaba una política ambiental que promovía la actividad extractiva del sector. ¿Cuáles son los argumentos del fallo judicial para rechazar la demanda de inconstitucionalidad? ¿Qué tipo de racionalidad construye el máximo tribunal sobre el ambiente? ¿Se trata de una visión contrahegemónica? ¿Cuáles son las determinaciones históricas del fallo judicial? Sin pretender abarcar la totalidad de estos interrogantes ni suponer que tienen una única respuesta, el presente trabajo se propone realizar una primera aproximación al análisis de la hegemonía discursiva del fallo judicial.

La lucha social contra la minería a cielo abierto tiene en Argentina una historia de la cual la provincia de Mendoza no ha sido ajena. Si la experiencia de Esquel, hacia el año 2002, marcó el punto inicial en el camino de visibilización nacional del conflicto megaminero, en Mendoza la conflictividad emergió entre 2003 y 2004 en la zona de Valle de Uco. A partir de allí se abrió en diferentes zonas de la provincia un período de movilización social que incluyó diferentes repertorios de acción (marchas, ayunos, cortes 
de rutas, actos, escraches, petitorios, charlas y debates, etc.). A través de esa experiencia de lucha se fue configurando, en forma amalgamada, la propia dinámica del conflicto, la constitución de los sujetos y colectivos involucrados y la formulación de sus demandas. ${ }^{1}$

El año 2007 es un punto de inflexión en este proceso, puesto que el ciclo de resistencia social abierto en diferentes zonas de la provincia (San Carlos, San Rafael, Alvear, Uspallata, Ciudad) logró imponer en la legislatura provincial la sanción de la mencionada Ley 7722, inscripción jurídica de la demanda social "el agua de Mendoza no se negocia”." Momento instituyente de la práctica política, social y cultural que las diversas asambleas y vecinos/as autoconvocados/as llevaron adelante en todo el territorio mendocino en defensa del agua como bien común (Onofrio et al. 2010).

A partir de allí, la ley 7722 devino uno de los asuntos clave en la disputa social y adquirió una particular relevancia en la dinámica del conflicto. Se advierte, en este sentido, el surgimiento de la arena legal-institucional como terreno de disputa y confrontación en el marco de las determinaciones del antagonismo social (Chinigioli, Barrientos y Fachinetti 2024), que fue cobrando particular importancia en la lucha contra la megaminería en la provincia. En realidad ese terreno de disputa está presente desde los comienzos del conflicto social respecto del ambiente por la megaminería, por ejemplo, en la experiencia de lucha de asambleístas en San Carlos, cuando parte del reclamo incluyó una ampliación de los límites de la Reserva Natural de la Laguna del Diamante (Ley Provincial No 7.422 de 2005). La encontramos también como parte de las demandas alrededor de las cuales, entre 2005 y 2006, se realizaron movilizaciones en el sur de la provincia, logrando sancionar diferentes ordenanzas municipales que establecían la prohibición de la minería metalífera (San Carlos, Gral.Alvear, Tunuyán, Tupungato), normativas que además incluían la promoción del agua como "bien común" y "recurso natural" (Onofrio et al. 2010). Con la promulgación de la ley 7722, ese terreno legal-institucional adquiere otros pliegues y significación política, ya que se trata de una normativa que regula la actividad en todo el territorio provincial y porque, además, abrió nuevos espacios y mecanismos de participación institucional e instancias de intervención social a las asambleas ciudadanas y otras organizaciones sociales. ${ }^{3}$

En suma, el fallo vino a concluir -siempre provisoriamente- un proceso de más de ocho años de judicialización de la disputa en torno a la megaminería. Así, el agua como bien común natural continúa siendo uno de los centros estructuradores del conflicto social provincial. La construcción pública del ambiente remite en el contexto provincial al discurso sobre el agua como recurso estratégico: "la manipulación histórica del agua constituye en Mendoza la principal determinación en torno de la cual se configura el conflicto ambiental” (Martín y Wagner 2013:315).

Ahora bien, el Estado provincial ha intervenido históricamente en la dinámica de esa conflictividad, involucrado en la construcción de "lo ambiental" como cuestión pública- 
social. En tal sentido, Martín y Wagner señalan que el Estado ha ocupado una posición irremediablemente contradictoria en cuanto al manejo tecnológico y político del agua: por un lado, ha recurrido a retóricas basadas en el imaginario ambiental mendocino -"cultura del agua"- pero, paradójicamente, ha sido a partir de ese imaginario que ha resultado cuestionada su política económica. En efecto, el Estado procura conciliar las actividades tradicionales de la provincia (industria vitivinícola) con la nueva actividad económica a la que apuesta (megaminería) desde un discurso basado en el desarrollo sustentable y el control ambiental (Martín y Wagner, 2013: 316). El lugar contradictorio de esa estatalidad es fundante de su enunciación discursiva e impregna el diseño de su política pública.

Aquí se trata de leer la sentencia como parte de una "apuesta social" que interviene en la construcción de hegemonía discursiva y se expresa a través de las referencias que circulan en el texto, organizando su sentido. Desde el punto de vista metodológico, he realizado una lectura crítica del texto del fallo, procurando hacer un seguimiento de las referencias que allí aparecen. Referencias diversas, pues remiten tanto a fuentes documentales como acontecimientos histórico-sociales, así como también a jurisprudencia y bibliografia específica. Para ello he tomado herramientas conceptuales provenientes del análisis del discurso. En tal sentido ha resultado crucial el aporte de Marc Angenot (2010) sobre el discurso social, para reconocer la profundidad de los vínculos que guarda el fallo con el discurso político y la construcción de hegemonía. Ese autor considera que cada época pone en circulación una oferta restringida de ideas, creencias y opiniones con predominancias, conflictos $y$ emergencias de sentidos que imponen límites históricos a lo pensable y posible de decir. El discurso social es todo lo que se dice y escribe en un determinado momento de una sociedad concreta, estableciendo repertorios tópicos y reglas de encadenamiento de enunciados que organizan lo decible. De alli que el fallo judicial de la SCJM, aún con las especificidades propias como dispositivo discursivo, constituye un valioso recurso para el análisis de la hegemonía discursiva, esto es, las maneras de conocer y representar lo conocido que conllevan determinadas apuestas sociales: "un conjunto complejo de reglas prescriptivas de diversificación de lo decible y de cohesión, de coalescencia, de integración" (Angenot 2012:24).

\section{El fallo judicial como apuesta social}

\subsection{La juridificación de los conflictos socioambientales}

La propuesta procura contribuir a la conceptualización de la disputa por los bienes comunes naturales, a través de un análisis centrado en el fallo de la SCJM como expresión de una determinada correlación de fuerzas y de ciertas estrategias seguidas por el bloque dominante para intervenir en la conflictividad social. Gabriela Merlinsky ha propuesto el término juridificación para nombrar una de las dimensiones de la "espiral del conflicto", análisis que implica considerar el proceso social mediante el cual los conflictos ambientales son "resignificados" cuando son llevados a la esfera del derecho (Merlinsky 2013:69). Incluye el análisis de la forma en que los actores utilizan el repertorio jurídi- 
co a su disposición, la intervención de los operadores del derecho en el conflicto y el ajuste de las expectativas de los actores sobre la ley y la justicia en general. Por su parte, Blichner y Molander advierten que esa juridificación se encuentra estrechamente relacionada con el inaudito desarrollo y expansión que tiene el derecho en las sociedades democráticas contemporáneas. ${ }^{4}$ Podemos considerar, para el caso de Mendoza, que tanto la promulgación de la Ley 7722 (2007) como el proceso de judicialización transitado desde entonces hasta el fallo aquí analizado (2015), constituyen expresiones relacionadas con una juridificación del conflicto megaminero.

La idea de una juridificación de los conflictos socioambientales permite entrever la profunda politicidad que impregna al discurso jurídico. Sus contornos se hacen visibles en diferentes ocasiones en la palabra de los actores aquí involucrados. Aparece, por ejemplo, de manera explícita en la letra del fallo -voto del Dr. Jorge Nanclares, por mencionar solo uno de ellos-, al reconocer que la SCJM estaba llamada a "resolver una cuestión pública que la política no resolvió”. Esa misma politicidad también es (d)enunciada -en forma de mensaje invertido- por el sector empresario minero afirmando que la SCJM "tenía que emitir un fallo totalmente técnico y no político" (Mdz, 17/12/2015), en un intento por desacreditar una decisión que resultaba claramente desfavorable a los intereses corporativos del sector. Efectivamente, hallamos a lo largo del documento judicial, enunciaciones que traman el hilo argumentativo acerca de la constitucionalidad de la ley y configuran, a la vez, un punto de vista sobre el conflicto abierto por la megaminería transnacional en la provincia. Podríamos decir que allí encontramos rastros de una ecología política -aunque como veremos no resulta totalmente homogénea, ni carente de tensiones-, cuya producción de sentido cobra una particular relevancia dado el carácter instituyente del Poder Judicial.

En primer lugar, hay en el texto una serie de giros recurrentes que dan cuenta de la disputa que está en juego, se trata de un reconocimiento que alude al profundo antagonismo social que enmarca la cuestión a dirimir. Así, podemos observar los siguientes pasajes:

Hoy se llama a resolver una cuestión pública que la política no resolvió, lo que debió ser una legislación producto del consenso y del diálogo social se convirtió en una controversia con fuerte participación de la sociedad, a veces hasta enfrentándola (Dr. Nanclares);

...no puede soslayarse que se está ante decisiones que la Provincia adopta en temas de enorme trascendencia por la hondura de los valores acerca de los que debe resolverse y su proyección con sentido histórico: Es necesario pensar que se corre el riesgo de afectar la matriz productiva y la preservación del recurso hídrico en medio de acechanzas 
a la biodiversidad, sólo por citar algunos de los aspectos involucrados (Dr. Gómez);

El debate que subyace a la problemática que aquí se plantea intenta superar un conflicto altamente complejo que involucra a varios sectores sociales (Dr. Palermo);

Entiendo que entorno a la Ley 7722 se encuentran en conflicto de bienes, intereses y valores colectivos, es decir, los recursos naturales, el ambiente, la sustentabilidad, el desarrollo económico, la actividad minera, entre otros. Esto provoca que los debates, discusiones y abordajes tengan un gran impacto en lo institucional, político, social, económico y cultural; con repercusiones no solo a nivel provincial, sino regional, nacional e internacional (Dr. Mario Adaro).

En segundo lugar, ese reconocimiento expresa también el grado de involucramiento del poder judicial y el rol específico que le corresponde, frente al cual el Tribunal afirma:

Es deber de los jueces desentrañar el contenido y alcance del derecho vigente, pero lo es más acompañar la realidad que les toca vivir (Dr. Nanclares);

Resulta fundamental la determinación del rol que debe cumplir el Estado -en general- y el Derecho -en particular-, como instrumento de la política, para determinar un equilibrio que permita un marco de sustentabilidad, sin perjudicar el resto de los intereses sociales implicados (Dr. Palermo)

la cuestión a resolver se presenta en términos de conflicto enmarcado en un debate de juridicidad constitucional, en que este Poder Judicial está llamado a intervenir declarando el derecho (Dr. Gómez)

Esto nos obliga a todos los ciudadanos-habitantes, pero en especial a los poderes públicos, y en mi caso, por la función que me ha sido asignada; a proteger el medio ambiente, lograr la utilización de los recursos naturales renovables desde el desarrollo sustentable y la prevención de daños al ambiente, minimizando el impacto de las actividades humanas (Dr. Adaro).

En efecto, el texto se encuentra surcado por una particular preocupación: interpelar a la “ciudadanía”. Por ejemplo, el Dr. Mario Adaro afirma explícitamente: 
mi primer objetivo es intentar expresar mi posición de una manera clara $y$ sencilla, $y$ de accesible comprensión por todo habitante - ciudadano - justiciable, aún advirtiendo que desarrollaré capítulos de abordaje técnico-jurídicos complejos que no pueden ser obviados.

El grado de involucramiento, en ocasiones, avanza incluso más allá de las funciones delimitadas por la "división de poderes", aportando ideas que se orientan en un sentido propositivo:

la alternativa es la construcción de formas e instancias estables de concertación, en las que sea posible la participación permanente de los diferentes actores o sectores involucrados, en instancias de diálogo, de saberes y conocimientos técni$\cos y$ científicos, en el despliegue de responsabilidad social ambiental y empresaria, en el respeto a las instituciones $y$ sus competencias, y la confianza social en ellas tendiendo a lograr una convivencia y democrática (Dr. Adaro); cada sociedad define, decide y gestiona el modelo que considera más conveniente para el desarrollo productivo (...) En consecuencia, aquella definición constituye una cuestión política que no puede reducirse al análisis puramente jurídico y que, para su reversión, es necesaria una actividad de política pública que desarticule la polarización existente entre los sectores en pugna desde un ámbito externo al estrictamente jurisdiccional (Dr. Palermo).

El conjunto de estos recortes procuran sostener la hipótesis del fallo judicial como parte de una apuesta social en la construcción de hegemonía; lectura que, entiendo, es de alguna manera ratificada por el resto de los sectores sociales, ya que ha sido exactamente en esa misma medida como ha sido recibida. Por ejemplo, Diego Seoane, uno de los diputados autores del proyecto de la ley presentado en la legislatura, destaca en una entrevista el compromiso demostrado por el poder judicial: "el fallo tiene un contenido muy profundo, los jueces se han comprometido con el tema" (Unidiversidad, 17/12/2015). Por su parte, el presidente de la CAMEM (Cámara de Empresarios Mineros Mendocinos) denunciaba, al momento de conocerse el fallo, que tenía "mucho tinte político"; mientras que Raúl Rodríguez, abogado de las empresas mineras demandantes, en una entrevista para el diario El Sol, hacía una observación en la misma dirección, "han hecho una valoración extrajurídica":

La Corte entregó una visión de la actividad minera que excede lo solicitado. No se ha merituado la prueba y se hace referencia a situaciones que no han sido parte del 
juicio. En la presentación no pedimos un pronunciamiento judicial sobre la minería, no le consultamos si es mala o es buena. Lo que solicitamos es que se pronunciara si esta ley era o no constitucional, pero la Suprema Corte hizo un análisis social, político y mediático de la minería, cuando nosotros fuimos a discutir una cuestión de puro derecho (El Sol, 17/12/2015).

Por su parte, una integrante de la Asamblea Mendocina por el Agua Pura afirmó:

Es lo que esperábamos, no había otra alternativa: la Ley 7722 es constitucional, no solo desde lo técnico sino también desde lo social, desde lo político. Es una ley que es una respuesta a los reclamos de la ciudadanía. Y si las leyes no están hechas para los ciudadanos, ¿para quiénes están hechas? Si las leyes no respetan la voluntad popular, entonces ¿para qué o para quiénes están hechas? (Los Andes, 16/12/2015b).

\subsection{La ambientalización del derecho}

Los fragmentos anteriores ponen de manifiesto la profunda politicidad que invade la sentencia, vehiculizando una mirada singular sobre la cuestión ambiental. Si he referido al proceso de juridificación de los conflictos socioambientales, también es significativo considerar su reverso, la ambientalización del derecho. La idea de un proceso histórico de ambientalización describe la manera como el asunto del "ambiente" ha cobrado relevancia histórica y ha sido construido como asunto de debate en el espacio público. José Sergio Leite Lopes lo describe como el desarrollo histórico de largo plazo -principios de los años setenta- por el cual se produce la invención, consolidación y avance de la temática ambiental en las sociedades contemporáneas. Desde el punto de vista de este autor, ello se relaciona con la construcción de una nueva cuestión social, esto es, la preservación o el cuidado del medio ambiente como cosa pública (Leite Lopes 2006:34). Esa ambientalización de la cuestión social impregna el discurso de diferentes sectores y ámbitos sociales, tales como la política pública; el desarrollo del derecho; el propio sector empresario ("responsabilidad social empresaria"); o el campo religioso, donde ha interpelado a la estructura eclesiástica (segunda encíclica del Papa Francisco, Laudato Si). Así, el proceso de ambientalización implica transformaciones en el Estado y en el comportamiento de las personas en la vida cotidiana.

En el fallo de la Corte, ese proceso de ambientalización de la cosa pública impregna todo el documento: 
Nos hallamos ante el surgimiento de un nuevo bien jurídico colectivo: el ambiente" (Dr. Nanclares);

El bien jurídico a tutelar: el derecho a un ambiente sano como Derecho Humano (...) La protección y la tutela ambiental constituyen un problema prioritario y ocupa un lugar cada vez más importante dentro de la agenda política nacional e internacional (Dr. Adaro);

El ambiente se ha transformado en un recurso crítico: si antes parecía infinito, inagotable, ahora hay conciencia de que es escaso (...) se declaró el derecho humano al ambiente adecuado para vivir en dignidad y bienestar y el consecuente deber de protegerlo y mejorarlo (Dr. Gómez).

Los propios magistrados ofrecen en el documento un relato acerca de cómo ha sido, desde su visión, ese proceso de ambientalización en las instituciones del derecho:

El original pacto federal que contiene nuestra Constitución Nacional de 1853/1860 (...) hizo silencio respecto de las cuestiones ambientales cuya incidencia en las necesidades de regulación y construcción autónoma por la ciencia jurídica aún no habian sido detectadas (Dr. Gómez);

Su explicación se encuentra en tanto la relación del hombre y el medio ambiente no se encontraba amenazada, no existía una preocupación por el ambiente hasta tanto no hubo una necesidad inmediata, tangible y preocupante (Dr. Adaro);

(L)a cuestión se mantuvo incluida en parámetros de legislación y reglamentación propios de otras materias (derecho administrativo, penal, civil, minero, laboral, etc.) (Dr. Gómez).

A partir de la década de 1970

la comunidad internacional ha tomado conciencia de que el desarrollo económico provoca deterioro en los recursos naturales y que éstos no son ilimitados sino que son finitos (...) Esto ha implicado redefinir la relación jurídica entre el hombre- el entorno y el derecho. En especial, ha sido necesario introducir importantes cambios en numerosas instituciones jurídicas (Dr. Adaro); 
Las propias previsiones de la Constitución Nacional reformada en 1994 y los marcos de convencionalidad que como consecuencia de la Constitución y los tratados fueron imponiéndose, promovieron un sistema de legislación propio de la materia ambiental (Dr. Gómez).

Ese relato acerca de la irrupción de lo ambiental como nueva cuestión social, cosa pública, puede ser entendido desde el pensamiento crítico como parte de una estrategia que, a la vez que reconoce un problema, ofrece una determinada forma de tratar el mismo. José Seoane advierte que la emergencia de la "cuestión ambiental" remite a un proceso ambivalente, pues está profundamente marcado por un contexto histórico particular, la frustración del ciclo de conflictividad global de los años 60 y la tendencia a la consolidación del neoliberalismo a nivel mundial. Así, a la vez que supone el reconocimiento de una problemática global (la crisis socioambiental), se trataría más bien de una "invención sociohistórica" que ha tendido a construir lo ambiental como parte de una estrategia política global que apuesta a procesar de una manera no antagónica las contradicciones sociales del capitalismo;

En ese sentido, la emergencia y configuración de la cuestión ambiental en este período puede ser entendida como parte de este tratamiento dominante del ciclo de rebelión global, particularmente referido a los cuestionamientos, programáticas y prácticas vinculadas a la disputa sobre las condiciones de existencia y reproducción de la vida social. La invención de la cuestión ambiental puede considerarse así, simultáneamente, uno de los agentes y uno de los resultados de esa frustración. Simultáneamente, emergencia y gestión sistémica de las contradicciones sociales desplegadas en esos años (Seoane 2017:43).

Este apartado ha tenido como finalidad presentar aquello que resulta crucial para pensar el fallo de la SCJM como parte de una estrategia en la construcción de la hegemonía discursiva alrededor de la cuestión ambiental, esto es, el profundo vínculo con lo político. La noción de juridificación contribuye al reconocimiento de algunos de los pliegues que adquieren esas vinculaciones del poder judicial con la dinámica del conflicto social en torno de la megaminería en Mendoza. La propia letra del documento da cuenta de la insistencia con la que se presenta: impone a los Magistrados el reconocimiento del grado de disputa y la trascendencia del asunto a dirimir (aquello que está en juego, el “destino de Mendoza”), frente a lo cual el poder judicial no puede sino sentirse convocado a intervenir desde lo que entienden su rol específico. Ahora bien, el texto de la sentencia expresa, a su vez, las determinaciones paradójicas que recibe de aquel vínculo con lo político. El fuerte antagonismo social configurado alrededor de la megaminería está ahí socavando en forma permanente la argumentación jurídica, al menos como 
suele entenderse -supuesto código objetivo-, incluso entre profesionales de dicho campo -por ejemplo si recordamos la definición del abogado de las empresas mineras, que refiere el problema a "una cuestión de puro derecho"-.

En efecto, la fuerte disputa social alrededor de la megaminería transnacional asoma insistentemente, a modo de una "protuberancia", en cada uno de los votos de los magistrados; pero lo hace sin que pueda ser integrado totalmente al propio cuerpo del fallo. Así, lo político adquiere la forma de un asunto al que resulta imprescindible aludir, aunque sea de manera paradójica, puesto que a la vez que lo "degrada", le otorga congruencia y hace inteligible. Está allí presente para producir una especia de exceso. De hecho, la demanda de inconstitucionalidad es considerada la "última ratio" en términos jurídicos, aunque en el tratamiento que le da CSJM hay un generalizado intento por sustanciar cada voto recurriendo a un abordaje más amplio que el exclusivamente técnico-jurídico. Este es otro rasgo a partir del cual es posible reconstruir fragmentos de ecología política, pues el texto del fallo nos entrega una visión de la actividad minera.

En suma, la apuesta por interpelar a la ciudadanía, la necesidad de ofrecer un relato acerca de los orígenes del derecho ambiental, el recuso a diversos materiales para justificar su decisión, incluso el avanzar sobre ideas propositivas acerca del procesamiento del conflicto en cuestión (a favor de una política del "diálogo"), son elementos del discurso social que contiene y transmite el fallo judicial. Si tal como afirma uno de los magistrados, se trata de "una cuestión pública que la política no resolvió", esto no quiere decir otra cosa que el hecho de que estamos frente a un conflicto que permanece abierto que, a la manera de una fuerza centrífuga, atrae y absorbe pero también desborda el terreno estrictamente jurídico.

\section{Referencias cruzadas}

En la sentencia plenaria sobre la constitucionalidad de la Ley 7722 el máximo tribunal provincial elaboró un texto de aproximadamente 70 páginas. De los siete jueces intervinientes, ${ }^{5}$ solo en uno de los casos el voto no fue debidamente fundamentado. ${ }^{6}$ A lo largo de todo el documento aparece una serie de referencias heterogéneas pero también recurrentes y que gravitan de manera transversal a toda la sentencia; a esa búsqueda está dedicado este apartado. Cabe aclarar que el seguimiento realizado no supone la exhaustividad de las referencias, ni el orden de su exposición algún tipo de jerarquía.

\subsection{Los metales pesados de una coyuntura}

En primer lugar cabe destacar la relevancia que adquieren en la sentencia ciertos acontecimientos históricos, en particular el derrame de solución cianurada producido en Veladero, explotación minera perteneciente a la canadiense Barrick Gold, ubicada en la ciudad de Jachal (San Juan, Argentina); y la rotura de los diques de contención de residuos mineros de la empresa Samarco Mineração S.A. (emprendimiento conjunto entre 
Vale S.A. y BHP Billiton) en Mariana (Minas Gerais, Brasil). Ambos acontecimientos irrumpieron en el espacio público provocando un fuerte debate social y mediático hacia finales de 2015, precisamente cuando el proceso judicial tendría su desenlace.

La importancia de estas referencias se desprende del lugar que ocupan en el fallo: cada una aparece en la sustanciación mediante la cual algunos magistrados convalidan los artículos más cuestionados por parte de las empresas mineras. Por un lado, el artículo primero, que establece la prohibición de ciertas sustancias tóxicas en la explotación de la minería metalífera. En su consideración, el voto del Dr. Nanclares alude al derrame en Veladero para dar "prueba" del "potencial daño" asociado a la megaminería y afirmar que -desde el punto de vista jurídico- el "principio de igualdad" no es absoluto sino que puede suponer también el reconocimiento de diferencias si son "razonables":

Evidentemente el criterio seguido por el legislador para hacer el distingo prohibiendo determinadas sustancias químicas es por el potencial daño que ellas representan".

Por otro lado, el artículo tercero, instituye el requisito de que la Declaración de Impacto Ambiental sea ratificada mediante ley por la legislatura provincial. En tal sentido, el voto del Dr. Perez Hualde, acuerda la "razonabilidad” de esa disposición afirmando que

los hechos -de por sí solos- constituyen una severa advertencia y una habilitación sobre los niveles de seguridad que cada provincia puede legítimamente adoptar cuando se trata de su territorio y de sus responsabilidades políticas frente a su pueblo y a su futuro.

El derrame en Veladero ocurrió el 15 de septiembre de 2015 y el de Samarco el 5 de noviembre del mismo año, ambos poco tiempo antes de la resolución de la SCJM sobre la ley 7722. La contaminación del río Jachal por el primer derrame en Veladero ${ }^{7}$ marcó particularmente el debate en Mendoza. En primer lugar, por la proximidad con esta provincia. El registro en los diarios locales daba cuenta de ello: "La duda que existe es si la presión social producto de lo ocurrido en la vecina provincia de San Juan puede afectar la decisión de los jueces, ya que el derrame se registró en un momento especialmente sensible para la provincia” (El Sol, 11/10/2015); "El reciente episodio sucedido en la mina Veladero en San Juan habría sido uno de los factores determinantes en la decisión del máximo tribunal mendocino" (Los Andes, 16/12/2015a).

En segundo lugar, por el particular lugar que ha ocupado históricamente San Juan en la construcción de la narrativa dominante sobre megaminería a nivel nacional, caballito de batalla del poder semiótico del capital. En este sentido, afirma Mirta Antonelli, el 
derrame en Veladero ha funcionado como implosión del núcleo duro del modelo de la megaminería metalífera en Argentina.

Este desastre ha hendido el corazón mismo del dispositivo de alianza Estado-corporaciones en Argentina al producir la incontrolable rasgadura de la profecía perversa del «desarrollo por la minería sustentable y responsable», y exhibir lo obsceno, lo fuera de escena que compromete la credibilidad del Anuncio férreamente sostenido: el destino minero como «redención de un pueblo postergado» (Antonelli 2016:60).

De la misma manera impactó en el debate público la avalancha de lodo tóxico que se produjo al romperse los muros de contención de las represas Fundão y Santarém, ambas ubicadas en la localidad de Bento Rodrigues, a 35 kilómetros del centro de Mariana (Minas Gerais). La corriente de residuos mineros destruyó la cuenca del rio Doce, eliminando toda la vida acuática y sepultando un pueblo entero. Los diarios locales de Mendoza registraron el acontecimiento como una verdadera "tragedia" (Los Andes, 6/11/2015), el “mayor desastre ambiental” de Brasil (El Sol, 25/11/2015). La dimensión de la destrucción socioambiental de ese hecho marca

(el) "advenimiento de una falla persistente entre la dinámica de la acumulación de capital y la reproducción ecológica de la vida. Un fenómeno estruendoso y local de un proceso de colapso civilizatorio más general” (Alimonda 2016:1).

En síntesis, ambas referencias abren una brecha en la "narrativa promesante" sobre la minería responsable, con control del Estado y funcional a un desarrollo sustentable que se intenta construir desde los sectores dominantes. Estos acontecimientos irrumpen en el espacio público fijando ciertos "límites y presiones" -tal como pensaba Raymond Williams (2000) que se ejercían las determinaciones históricas- al debate ideológico y las condiciones de (in)visibilidad sobre megaminería transnacional.

\subsection{Del peso de lo jurídico y la ceguera de la justicia}

Un segundo grupo de referencias que encontramos en el texto del fallo judicial se trata, por supuesto, del corpus documental compuesto por la jurisprudencia y la teoría del derecho, principalmente vinculada a la rama del derecho ambiental. En efecto, encontramos en el trabajo de sustanciación de la mayoría de los votos una revisión de la bibliografia especializada en derecho ambiental y la legislación positiva. Al respecto, el Tribunal fijó el tratamiento de la ley 7722 en una doble dirección. Por un lado, el análisis de la constitucionalidad, en el cual resultan como lugares comunes el análisis de la reforma constitucional de 1994, principalmente la centralidad del artículo 41 de 
“presupuestos mínimos”; el Código de Minería, la Ley General del Ambiente 25675, entre otros. Por el otro, el control de convencionalidad, revisando el conjunto de tratados internacionales a los que Argentina adhiere otorgando jerarquía constitucional, entre los que se destaca la Convención Interamericana de Derechos Humanos. ${ }^{8}$

De la misma manera, hay en el texto de la sentencia un análisis de la jurisprudencia, tanto de la doctrina establecida por los órganos judiciales nacionales (Corte Suprema de Justicia de la Nación) como también internacionales (Corte Interamericana de Derechos Humanos). Entre los precedentes de mayor gravitación, encontramos el "caso Villivar" (C.S.J.N., Fallos 330:1791, Sentencia del 17/04/2007) originado en el recurso de amparo que interpuso Silvana Villivar, joven vecina de Esquel, en diciembre de 2002 contra la Minera El Desquite S.A., subsidiaria de la canadiense Meridian Gold (adquirida luego por Yamana Gold); el cual lograría suspender la actividad de la minera a raíz de la inexistencia de una audiencia pública previa a la aprobación de la Declaración de Impacto Ambiental, tal como estipulaba la legislación provincial. Otro fallo citado por más de un magistrado, es el caso "Beatriz Mendoza", en referencia a la demanda judicial que presentaron vecinos/as de la localidad de Avellaneda (provincia de Buenos Aires) por la contaminación de la cuenca Matanza-Riachuelo.

Es crucial señalar la importancia de ambos casos en la irrupción del ambiente como problema social. De hecho, ambos se encuentran entre los cinco hitos que, según Maristella Svampa y EnriqueViale, marcaron “una inflexión en el modo en cómo se piensa, se tematiza y se debate la cuestión ambiental en la Argentina” (Svampa yViale 2014:62). En el caso Villivar, vinculado estrechamente a la regulación de la megaminería, la Corte Suprema de Justicia de la Nación dirimió la competencia que tienen las provincias de complementar las normas que contengan los presupuestos mínimos de protección del medio ambiente (art. 41 de la Constitución Nacional). La significancia de ese caso se vincula a lo que Maristela Svampa ha denominado el "efecto Esquel” (Svampa 2009), el primer conflicto que irrumpe en la arena nacional, permitiendo la visibilización de la lucha social contra la megaminería transnacional y de resultado exitoso, de allí su arrastre multiplicador sobre otras regiones del territorio nacional y experiencias de resistencia frente a proyectos de minería a gran escala. El segundo precedente, el caso Beatriz Mendoza, constituye un punto de inflexión político-institucional en el tratamiento ambiental en Argentina, puesto que la configuración del conflicto incluyó las relaciones entre diferentes jurisdicciones de gobierno (nacional, provincial, local) y la responsabilidad que le cabe a una diversidad de empresas e industrias en la contaminación y degradación del territorio sobre el que operan (Merlinsky 2009). Por último, encontramos la sentencia del Tribunal Superior de la Provincia de Córdoba sobre la constitucionalidad de la Ley 9526 -otras de las "leyes del no" como se ha llamado a las normativas provinciales que interpusieron un freno al avance de los proyectos de megaminería transnacional en Argentina-. ${ }^{9}$ Este fallo "relativo a la misma problemática" (expte. n 1798036 
in re "Cemincor") es del día 11 de agosto de 2015, es decir, apenas unos meses antes del fallo judicial mendocino para el cual sentó un importante precedente.

En contraste con este corpus relativo al derecho, cabe destacar la escasa presencia de bibliografia especializada proveniente del amplio campo de las ciencias sociales, humanas y ambientales. Esto es algo que llama la atención al menos por dos motivos. En primer lugar, debido a la abundante producción que sobre la temática han aportado una variedad de disciplinas científicas durante la última década en Argentina y toda la región latinoamericana. Una abundante bibliografia relativa a estudios realizados desde la economía, sociología, ciencias políticas, historia, antropología, ecología política, etcétera. En segundo lugar, porque dicha ausencia contrasta no solo con el peso relativo de la jurisprudencia y la teoría del derecho, sino también con la vocación que hace explícita la SCJM de fundamentar su decisión en una visión ampliada del problema ambiental; esfuerzo que por otra parte es reiteradamente demostrado al recurrir a perspectivas por fuera de lo estrictamente jurídico, como por ejemplo, la encíclica papal Laudato Si.

Aquí no sorprende la inclusión de la segunda encíclica papal dentro del marco de referencias que sostiene el fallo, puesto que la misma ha promovido un sustantivo aporte al debate económico, social y cultural que es necesario frente a la crisis ambiental hoy en curso. Algo que ha sabido destacar Antonio Elizalde respecto de los documentos papales Laudato si y Evangelii gaudium como aportes al pensamiento y acción de un modo de vida sustentable. En dichos documentos, Francisco "recoge gran parte, sino toda, la reflexión que desde América Latina se ha venido haciendo en torno a los problemas de la sustentabilidad y de la justicia social" (Elizalde 2015:145).

Lo que llama la atención es la ausencia de referencias al conocimiento científico producido en y desde las ciencias sociales y ambientales. Esa exclusión, cabe destacar, no resulta sin efectos en la perspectiva ambiental que construye el máximo tribunal provincial, algunos de los cuales es posible reconocer en asuntos que resultan clave para el debate en cuestión. Uno de ellos es la necesidad de diferenciar entre minería tradicional (subterránea) y megaminería o minería a gran escala. Desde momentos tempranos se advirtió en el debate público sobre la minería transnacional, acerca de la necesidad de diferenciar a qué tipo de minería nos referimos cuando hablamos de minería a gran escala:

(...) lejos estamos de aquella minería de socavón, propias de otras épocas, cuando los metales afluían en grandes vetas, desde el fondo de las galerías subterráneas. En la actualidad, los metales, cada vez más escasos, se encuentran en estado de diseminación y sólo pueden ser extraídos a través de nuevas tecnologías (...). En suma, lo particular de este tipo de minería (a cielo abierto), diferente de la 
tradicional, es que implica niveles aún mayores de afectación del medio ambiente, generando cuantiosos pasivos ambientales, al tiempo que requiere tanto un uso desmesurado de recursos -entre ellos el agua y la energía, imprescindibles para sus operaciones- como la intervención de manera violenta en la geografía de los territorios para la explotación (Svampa y Antonelli 2009:16)

En un documento de más de 70 páginas como es el que contiene la sentencia que estamos analizando, la palabra "megaminería" (de un uso hoy ampliamente extendido) no aparece ni una sola vez. La misma ausencia registran las denominaciones "a gran escala" o "transnacional", mientras que -por el contrario- términos como "minería" y "actividad minera" suman entre ambas 117 entradas. Por su parte, la especificación de minería "a cielo abierto" aparece solo en dos ocasiones: la primera, en relación a un precedente -citado como fallo "Cemincor"-, mientras que la segunda tiene lugar -no casualmente- a partir de la única excepción que podemos encontrar a lo que se viene señalando, correspondiente a una cita de Bellotti (2011:391-437). Soslayar esa distinción es producir un borramiento de las muy diferentes consecuencias socioambientales que tienen disímiles tipos de explotación minera, es decir, se trata de una profunda deshistorización del conflicto y las prácticas sociales en juego.

El discurso que organiza y sostiene el fallo judicial resulta así envuelto en categorías abstractas y ahistóricas (tales como "actividad minera", "escasez", "riesgo") y susceptible de quedar atrapado en oposiciones binarias como las que se construyen en otras formaciones discursivas, por ejemplo, el discurso periodístico que ha tendido a organizar el debate sobre la base de una oposición dilemática minería/antiminería (Salomone 2017). No es casual entonces que podamos observar a lo largo del fallo judicial posturas e ideas que se vinculan, directa o indirectamente, al "imaginario prominero" que desde el pensamiento crítico se intenta desmontar en torno de la megaminería y el llamado extractivismo en general (Voces de alerta 2011), entre esos mitos cabe destacar la minería como "motor del desarrollo y el crecimiento económico", o el "destino mineral" para nuestras regiones cordilleranas.

\subsection{El Estado ecológico del derecho}

Un último conjunto de referencias que permite rastrear fragmentos de una ecología política subyacente al fallo judicial, tiene que ver con la centralidad que adquieren en este documento las declaraciones e informes producidos en el marco de la Organización de Naciones Unidas (ONU). Algunos de los documentos que circulan con mayor gravitación en la sentencia son los siguientes: la Declaración de Estocolmo llamada "Nuestro Futuro en Común” (1972); el Informe Brundtland (1987); y la Declaración de Río (1992). Ese corpus documental está profundamente ligado al proceso de ambientalización 
mencionado más arriba; en particular, denota la especificidad que adquiere en la esfera global el tratamiento de la cuestión ambiental. Alrededor de esa serie de declaraciones se desprenden dos nociones que resultan cruciales para pensar la cuestión ambiental y ocupan un rol central en el fallo judicial el principio de precaución y el desarrollo sostenible.

Seoane señala que el recorrido que va de la Conferencia de Estocolmo (1972) a la Cumbre de la Tierra (como se conoce a la Conferencia de Río de 1992), expresa la profunda reconfiguración neoliberal que supuso el tratamiento internacional de la cuestión ambiental (Seoane 2017).${ }^{10}$ Es decir, a través de esas referencias, el fallo judicial incorpora y hace suya la racionalidad ambiental construida durante las últimas décadas a nivel internacional. A partir de esa hipótesis, entiendo que la impronta neoliberal introduce de alguna manera una importante tensión en la perspectiva del fallo judicial sobre la cuestión ambiental. Mientras el principio precautorio aparece como una concepción que permite en cierta medida y no sin ambigüedades, subvertir la jerarquía dominante, priorizando el cuidado del “medio ambiente" frente a los riesgos asociados a determinada "actividad económica", la noción de desarrollo sostenible, por el contrario, se liga más bien a una estrategia internacional que tiende a subordinar la cuestión ambiental al desarrollo entendido en términos clásicos, esto es, fundamentalmente como crecimiento económico sin fin.

\section{La defensa del bien común bajo el "principio precautorio"}

El principio precautorio resulta transversal a toda la sentencia de la SCJM, ocupando un lugar destacado en la mayoría de las visiones ofrecidas por sus integrantes.

El fallo en su conjunto afirma al principio precautorio como uno de los pilares del derecho ambiental. El Dr. Nanclares advierte que "En la lucha en defensa del ambiente y la salud se imponen los mecanismos anticipatorios, de tutela temprana, precoz, a la luz de los principios de prevención y precaución de política ambiental", mientras que el Dr. Palermo afirma: "la Ley 7.722 optó por resolver esta alternativa sobre la base de un principio fundamental del Derecho ambiental: el "principio de precaución»". En la misma dirección apunta el análisis de constitucionalidad que realiza Julieta Lavarello -abogada y militante de la Asamblea Mendocina por el Agua Pura- en su tesis de grado, quien resalta la importancia del principio precautorio en toda la legislación argentina (Lavarello 2016).

El principio de precaución, según Drnas de Clément (2008), tiene una genealogía de tiempo largo que se remonta al antiguo canon del comportamiento humano vinculado a la concepción de "prudencia" ante lo incierto, lo desconocido. Implica así una actitud de reserva, circunspección, previsión. Indisociablemente vinculado a la Filosofía del Derecho, el recorrido de este principio durante el siglo XX fue diverso, logrando diferentes tipos de inscripciones normativas. Mientras una tácita referencia a la precaución tuvo un amplio desarrollo a través de variados matices, el primer instrumento internacional de alcance universal general fue la Carta Mundial de la Naturaleza del 28 de octubre de 
1982; y la primera inclusión legislativa del contenido preceptual de la precaución a nivel nacional fue la incorporada en Alemania en 1971 (a través del término Vorsorgeprinzip).

El marco de referencia presente en la sentencia de la SCJM reenvía a la Declaración de Río de la Cumbre de la tierra. ${ }^{11}$ Este constituye el hito a partir del cual el principio de precaución logró un paulatino y sostenido fortalecimiento en un conjunto de acuerdos y tratados internacionales. Una primera característica que define al principio de precaución que es la incertidumbre científica. ${ }^{12}$ Resulta frecuente que el Derecho Ambiental deba operar con referencias y valoraciones inciertas o que están sujetas a controversias científicas. En dichas situaciones se hace prácticamente imposible no sólo cuantificar, sino conocer con una mínima precisión cuál será el daño causado al medio ambiente, sobre todo si se tiene en cuenta que en muchos casos sus totales efectos sólo se hacen perceptibles al cabo de un dilatado periodo de tiempo (Esteve Pardo 2006a). La precaución o virtud de la prudencia es un supuesto consustancial a todo el derecho, pero el principio precautorio tiene una funcionalidad propia y distinta a ese sentido general: la de fundar o habilitar una decisión de las autoridades públicas en situaciones de incertidumbre en el entorno y sobre los efectos mismos de esa decisión. En tal sentido, la hipótesis de la precaución nos pone en presencia de un riesgo no mensurable, es decir, no evaluable. En efecto, dos elementos resultan clave para situar la pertinencia de este principio en el derecho ambiental: el de la incertidumbre científica y el estar ante una situación de riesgo grave para el medio ambiente, es decir, cuando se trata de un daño irreversible (Rodríguez Salas 2004; Esteve Pardo 2006a). En el fallo judicial que analizamos dicha perspectiva es sintetizada por el Dr. Palermo, quien afirma:

Mediante este principio se pretende encontrar una máxima que ofrezca seguridad y control de riesgos en aquellas actividades que generan peligros y cuyas consecuencias son, todavía, científicamente desconocidas. En otras palabras, se recurre a este principio a fin de garantizar la seguridad donde domina la incertidumbre.

Otras de las características que hay que mencionar es la inversión que supone de la "carga de prueba": es quien genera el riesgo de daño grave e irreversible a la salud humana o al "medio ambiente" quien debe demostrar la inocuidad o seguridad de su actividad, o de la sustancia o producto que pone en el mercado. Ahora bien, es importante destacar que esta inversión también puede leerse en relación a una "ética" ambiental, puesto que permite de alguna manera subvertir la racionalidad económica dominante en nuestras sociedades: "El interés social siempre prevalecerá sobre los intereses económicos sectoriales” (Dr. Nanclares); “En definitiva, en primer lugar la protección humana y al ambiente, segundo los beneficios sociales de la actividad que se reflejen en la comunidad" (Dr. Nanclres); “el recurso al principio de precaución aparece como un «contraproyecto 
normativo» frente a la denominada sociedad del riesgo" (Dr. Palermo). De allí que el principio precautorio haya sido defendido por movimientos y organizaciones sociales que defienden bienes comunes naturales, puesto que constituye un posible freno al avance de la apropiación capitalista sobre sus territorios.

Como se sabe, en los extremos de esta discusión aparece lo que algunos describen como una antinomia entre la "permisión de la actividad minería» y la "preservación del recurso hídrico». Pues bien, si esta descripción es correcta, entiendo que la Ley 7.722 optó por resolver esta alternativa sobre la base de un principio fundamental del Derecho ambiental: el «principio de precaución» (Dr. Palermo).

El principio precautorio abre la posibilidad de oponer un límite a la racionalidad instrumental basada en la eficiencia medio-fin, que compulsivamente debe extender la relación costo-beneficio al máximo posible, aún a costa de producir una translimitación ecológica (Hinkelammert 2006).

Sin embargo, cabe señalar que aunque el principio precautorio permite esa apertura o inversión ética, no se contrapone necesariamente con la perspectiva del desarrollo moderno. Más aún cuando, precisamente, el principio precautorio encuentra en la Declaración de Rio lo que Zlata Drnas de Clément denomina una "formulación débil” (Drnas de Clément 2008:26), en sentido de que sólo "invita" a los participantes de la declaración a considerar la adopción de medidas cautelares -sin contener verdaderas obligaciones-, condicionando su aplicación a las capacidades de desarrollo del Estado y subordinando la amplitud del deber precautorio a la relación costo-beneficio de la medida. ${ }^{13}$ En tal sentido, el Dr. Nanclares afirma que "(el principio precautorio) no puede presentarse como un obstáculo al desarrollo del conocimiento sino por el contrario, debe ser el motor para el avance de la ciencia siguiendo el camino del desarrollo sustentable"; mientras que el Dr. Palermo advierte, "en una sociedad necesitada de tecnología e industrialización el principio de precaución tendrá un ámbito de operatividad mucho más reducido, pues la idea que subyace a este principio puede volverse disfuncional a su necesidad de desarrollo". La perspectiva del desarrollo sostenible pareciera estar allí para colorear el trasfondo sobre el que adquiere su particular significación el principio precautorio.

\section{El Desarrollo sostenible o la estructura de una concesión}

La noción de desarrollo sostenible es otro de los núcleos de sentido que organiza la sentencia de la SCJM que aquí analizamos. Es un concepto cuya genealogía remite a los debates que tuvieron lugar en el marco de las conferencias de Naciones Unidas a las que alude el fallo. En ese contexto, tuvo su primera aparición a propósito del Informe Brundtland (1987) e inmediatamente encontró su consolidación en la Declaración de Río (1992) 
como noción central en la definición de la cuestión ambiental. La Conferencia de Estocolmo (1972) constituye una inflexión a nivel internacional en la medida de que marca una primera intervención de Naciones Unidas sobre la cuestión ambiental. Su significación histórica ha sido sin dudas el haber promovido un tratamiento de lo ambiental como problemática global, acorde al proceso en curso de mundialización neoliberal. Mientras que la Conferencia de Río (1992) marca, a través de la consagración del desarrollo sostenible, una inflexión en el proceso de naturalización de la problemática ambiental; es decir, la creciente asimilación de la misma a la problemática del mundo natural (Seoane 2017).

En efecto, el marco de referencia de la ONU deja su impronta en el fallo analizado, en las significaciones que circulan acerca de la cuestión ambiental. Como señala Seoane, las trazas de esa racionalidad ambiental pueden reconocerse al menos en torno a tres características. Primero, la creciente utilización del término "medio ambiente", en contraposición a la idea de "medio humano" usado en la Declaración de Estocolmo, que hacía referencia al doble aspecto natural y artificial de los problemas ambientales. El generalizado uso del término "medio ambiente" es sintomático de la invención de lo ambiental como "mundo natural", cuestión que expresa la dualización sociedad/naturaleza propia de la colonialidad capitalista y patriarcal de la modernidad. Por ejemplo, el vocablo "medio ambiente" tiene un total de 83 entradas en el texto de la sentencia, de las cuales más de 60 refieren a una significación de lo ambiental claramente separada de la praxis histórica de los sujetos. Cuando la SCJM plantea la "protección del ambiente", se está refiriendo al agua, aire, paisaje, montañas, etc. Algo que se desprende del uso generalizado de "medio ambiente", como también a partir de los términos con los que muchas veces se acompaña la idea de ambiente: "preservación”, "protección” o "degradación” (33 instancias). Del mismo modo, en otras ocasiones, la alusión al medio ambiente hace explícita la distinción del mundo natural y artificial, refiriéndose al medio ambiente y... al "hombre", las "comunidades", los "pueblos originarios" o la "salud humana".

En segundo lugar, las huellas del pensamiento de la ONU sobre la cuestión ambiental se reconocen en una noción de "sostenibilidad" definida como compromiso intergeneracional. En alusión al Informe Brundtland, el Dr. Nanclares retoma la definición del desarrollo sostenible como "el desarrollo que satisface las necesidades del presente sin comprometer la capacidad de las generaciones futuras para satisfacer sus propias necesidades"; mientras que el Dr. Adaro afirma, a propósito de la idea de sustentabilidad, que se trata de "lograr un equilibrio entre la protección del medio ambiente, el progreso económico y el desarrollo social, sin comprometer el desarrollo de las generaciones futuras"; y el voto del Dr. Nanclares advierte "La tutela del ambiente no significa detener el progreso, sino por el contrario, hacerlo más perdurable en el tiempo de manera que puedan disfrutarlo las generaciones futuras".

Por último, podemos observar un especial cuidado por compatibilizar la cuestión am- 
biental -definida como mundo natural- con el crecimiento económico. Por ejemplo, se puede leer en la sentencia la afirmación de que

La minería representa una importante fuente de crecimiento económico para los países en desarrollo (...) Los minerales constituyen un punto estratégico para el desarrollo de la humanidad (Dr. Nanclares)

Resulta fundamental la determinación del rol que debe cumplir el Estado -en general- y el Derecho -en particular-, como instrumento de la política, para determinar un equilibrio que permita un marco de sustentabilidad, sin perjudicar el resto de los intereses sociales implicados (Dr. Palermo).

Desde el pensamiento crítico se ha señalado la profunda contradicción que supone el paradigma del desarrollo sostenible (Antonelli 2009, 2016; Alvater, 2011; Lander 2011; Svampa y Viale 2014; Seoane 2017). Más allá de los matices y posibles diferencias entre quienes lo cuestionan, hay un sentido común que apunta a señalar el hecho de que el desarrollo sostenible se revela como un paradójico intento de resolver los efectos de la acumulación capitalista -crisis ambiental, destrucción de los ecosistemas-, ampliando los márgenes de su dominio. Es decir, se trataría de un nuevo paradigma que en verdad no plantea nada por fuera de la lógica capitalista del crecimiento económico sin fin, de allí el "malabarismo conceptual" que debe operar para rejuvenecer la clásica noción de desarrollo y maquillar el afán de crecimiento económico ahora en concordancia con las leyes de la naturaleza. Quizás, lo más llamativo, es que dicha presentación la realiza con extraordinario éxito. Esa es su mayor virtud, la eficacia política e ideológica:

Respondió en términos que parecían tomar en cuenta los cuestionamientos al desarrollo, mientras que en realidad lo que hacía era reforzarlo. Operó como un dispositivo tranquilizador en la medida en que logró crear la ilusión de que se estaban tomando medidas efectivas en respuesta a la crisis diagnosticada. Al no cuestionar la lógica de la acumulación capitalista y el modelo de la sociedad industrial como causas fundamentales de la destrucción de las condiciones que hacen posible la vida, operó como mecanismo legitimador de la globalización neoliberal, que de ese modo pasó a presentarse como sostenible, a pesar de su avasallante dinámica devastadora (Lander 2011). 
En la misma dirección, Mirta Antonelli señala que el desarrollo sustentable funciona a la manera de una fórmula que se repite, migra, atraviesa discursos, géneros, instituciones, y con ello, logra una "alianza hegemónica" cuya pregnancia y eficacia sutura la narrativa extractiva. Un dispositivo transversal a las redes productivas, de gestión, de administración y promoción de discursos sociales, que incluye por supuesto los mensajes del Estado en sus múltiples instancias de enunciación, lugares desde los cuales contribuye a fijar los límites de lo decible y lo pensable (Antonelli 2009:55). Tal como afirma la autora, el desarrollo sostenible tiene la estructura de una concesión (Antonelli 2016:65), lo sustantivo es asegurar el desarrollo y luego que sea "sostenible" en el tiempo.

\section{Reflexiones finales}

La crisis global del capitalismo iniciada hace una década tendió a expresarse en la región latinoamericana como profundización y extensión del modelo extractivo exportador, esto es, como intensificación del proceso de acumulación por desposesión que reabrió un nuevo ciclo de mercantilización y apropiación privada de bienes comunes de la naturaleza. Esa ofensiva del capital resulta el telón de fondo sobre el que adquirieron visibilidad los "conflictos socioambientales" a inicios del nuevo siglo, movilizados por una diversidad de colectivos que se organizan en defensa de sus territorios y bienes comunes. En efecto, la centralidad que adquirieron los bienes comunes de la naturaleza en la conflictividad social es producto de esa tensión propia del capitalismo en esta fase histórica en la cual -como "salida" de la crisis global- hay un intento de recolonización de nuevos territorios y espacios sociales que coloca -una vez más- al ambiente, la naturaleza y la vida humana como centro del despojo y la devastación.

Esa ofensiva extractivista, hoy bajo nueva relegitimación neoliberal, no es posible sin transformar los territorios en espacios para la acumulación de capital. Para ello, tal como se ha señalado desde el pensamiento crítico, se requiere de un conjunto de dispositivos de intervención y estrategias de legitimación orientadas a naturalizar las relaciones sociales capitalistas. En este trabajo he propuesto un análisis de la sentencia de la SCJM sobre la constitucionalidad de la ley 7722 como parte de los dispositivos discursivos que se movilizan e intervienen en la construcción de una hegemonía discursiva, tal como es entendida por Marc Angenot, en tanto "apuesta social” (enjeux) orientada a definir, en un momento determinado, todo lo posible de decir y de penar, en este caso, el discurso social sobre lo ambiental. Es en ese sentido que podemos afirmar la existencia de una ecología política en el fallo judicial, documento que vehiculiza ciertas ideas, representaciones y significancias; en suma, una determinada trama ideológica en relación con la cuestión ambiental y la megaminería en particular.

La tarea supuso rastrear las huellas de esa ecología política que se expresa a través de las referencias más importantes que podemos encontrar en el texto de la sentencia. La 
relevancia de esas referencias estuvo determinada desde el punto de vista metodológico por lo que podríamos llamar un "muestreo intencional", esto es, una selección teóricamente informada. Así, el primer apartado estuvo orientado a reconocer la politicidad que impregna toda la sentencia y contribuye a revelar el lugar de enunciación de la SCJM como agente del discurso social. En tal sentido, se destaca que no se trata solo de una intervención que contribuye a organizar lo decible y pensable, los lugares comunes de la hegemonía discursiva, sino que muestra una preocupación por proveer y dotar a ese discurso de "aceptabilidad y encanto" (Angenot). Lo que organiza el discurso de la SCJM es el deseo de interpelar a la ciudadanía. Los conceptos de juridificación y ambientalización contribuyeron a determinar el profundo vínculo que guarda el fallo con la disputa social que tiene lugar en torno de los bienes comunes naturales.

El segundo apartado estuvo dedicado a exponer el análisis del marco de referencias presente en dicho texto. Referencias heterogéneas a través de las cuales el poder judicial construye una visión sobre la cuestión ambiental y en particular sobre la minería a gran escala, pues cada una de esos materiales que son históricos, documentales, religiosos y jurídicos, reenvía a un debate, a una determinada problematización y gestión de lo ambiental, a una particular mirada sobre la relación sociedad-naturaleza, etc.

Ahora bien, hemos visto que no se trata de una visión homogénea y unívoca. Más bien lo que encontramos es un marco de referencia fragmentario y contradictorio, en tensión. Pues así como los derrames producidos en Veladero (San Juan) y Samarco (Minas Gerais) resultan referencias cruciales dentro del fallo ejerciendo límites y presiones sobre la matriz que regula las condiciones de (in)visibilidad e (in)decibilidad alrededor de la megaminería; la referencia al principio precautorio se ve teñida por el paradigma del desarrollo sostenible que ha sido señalado, desde el pensamiento crítico, como uno de los dispositivos discursivos política e ideológicamente más eficaces a la hora de construir un blindaje a la política extractiva. En efecto, la defensa del agua como bien común, en el contexto de este fallo judicial, queda al amparo de una mirada, por lo menos, ambivalente. Ratifica la constitucionalidad de una ley que protege el ambiente y el agua, pero lo hace desde una perspectiva que no es inequívocamente incompatible con proyectos extractivistas y la política predatoria del capitalismo. El fallo reproduce por medio de sus referencias, buena parte de los elementos propios de la racionalidad ambiental hegemónica: la noción de progreso, el crecimiento económico sin fin, productivismo, antropocentrismo, ilusión tecnocrática, etc.

La defensa del agua que llevan a cabo las asambleas, vecinos/as autoconvocados/as y otras organizaciones sociales en Mendoza, ha logrado a través de la lucha social, poner un freno a los proyectos de minería contaminante sobre el territorio provincial. No obstante, se trata de un resultado provisorio, sujeto a la iniciativa de los sectores dominantes. Las empresas mineras ya iniciaron el recurso de apelación a la Corte Suprema de 
la Nación; mientras que el ejecutivo provincial, durante 2016, puso en marcha el Espacio de Diálogo Minería para un Desarrollo Sustentable, un claro esfuerzo y apuesta del gobierno provincial por reintroducir el debate sobre la megaminería a través de un tono discursivo que procura acomodarse al reciente fallo judicial: una minería limpia y responsable, sustentable, con mayor regulación estatal, etc. Las tensiones presentes en la sentencia de la SCJM ponen de manifiesto el sentido abierto de la política pública, el Estado como contradicción, la estatalidad como lugar siempre sujeto al devenir de la lucha de clases. El fallo de la Suprema Corte provincial es un documento cruzado por los conflictos que "la política no resolvió" y, como tal, los (contra)sentidos que construye sobre la cuestión ambiental reciben las marcas de esa profunda disputa societal que tiene lugar alrededor de los bienes comunes naturales. Cuál de esos sentidos prevalezca es una pregunta que el futuro de la lucha social deberá responder. 
${ }^{1}$ Un análisis de los comienzos de la lucha contra la megaminería en Mendoza puede encontrarse en Sergio Onofrio (2009), mientras que el trabajo de Lucrecia Wagner (2014) proporciona una amplia reconstrucción del proceso de provincialización del conflicto y sus vicisitudes. Existen estudios que analizan cuestiones particulares del conflicto, como la tesis de María Julia Barrientos sobre la "audiencia pública" del proyecto San Jorge en Uspallata (2012).

${ }^{2}$ Por supuesto, la inscripción jurídica de una demanda social no se realiza de forma lineal, sino que supone la introducción de una serie de torsiones, no solo por el trabajo de traducción que implica el desplazamiento por terrenos sociales diferenciados -el pasaje de la lucha callejera al ámbito de judicial-, sino por la heterogeneidad que habita al interior del propio movimiento social.

${ }^{3}$ A partir de 2008 las asambleas fueron invitadas a participar del Consejo Provincial del Ambiente (órgano asesor del poder ejecutivo que en verdad había sido incorporado por la Ley 5961de 1992); mientras que en 2011 cobró una particular importancia la participación ciudadana en el proceso de audiencia pública convocado por el proyecto San Jorge en Uspallata. Participación que culminó con las contundentes movilizaciones en la legislatura provincial en rechazo a dicho proyecto, el mismo día que debía ser tratada la aprobación de la EIA (Evaluación de Impacto Ambiental) para ser ratificada por ley, tal como establece precisamente el artículo tercero de la ley 7722.

${ }^{4}$ Estos autores relacionan la juridificación con cinco tendencias presentes en nuestras sociedades (Blichner y Molander 2008:38-48): a) ciertas reformas políticas cuyos efectos suponen incorporar mayores competencias a la esfera legal; b) la expansión y diferenciación de la ley y el derecho que supone una aplicación progresiva de normas legales a un amplio rango de temas; c) una creciente resolución de los conflictos por medio de recursos legales (referencialidad de la ley); c) una creciente resolución de los conflictos por medios legales (referencialidad de la ley) d) el incremento del poder judicial, la creciente influencia social de los tribunales y de los profesionales del derecho; e) la existencia de marcos jurídicos como referencias dominantes en la configuración de los vínculos interpersonales. ${ }^{5}$ El orden de los votos es el siguiente: primero Dr. Jorge Horacio Nanclares; segundo; Dr. Herman Amilton Salvini; tercero: Dr. Julio Ramon Gómez; cuarto: Dr. Omar Alejandro Palermo; quinto: Dr. Alejandro Perez Hualde; sexto: Dr. Mario Daniel Adaro; séptimo: Dr. Pedro Jorge Llorente.

${ }^{6}$ El Dr. Hernán Amilton Salvini simplemente adhirió a la fundamentación provista por su antecesor, Dr. Jorge Nanclares. ${ }^{7}$ Con posterioridad han sido de público conocimiento al menos dos derrames más.

${ }^{8}$ El convencionalismo es "entendido como el acto de control que efectúa el juez en cuanto a la conformidad de la norma interna a la Convención Americana sobre Derechos Humanos" (voto Dr. Mario Adaro). A partir del fallo "Ekmedjian v. Sofovich" (CSJN, Fallos: 315:1492), el Máximo Tribunal de la nación Argentina comenzó a tomar como guía, en especial en materia de derechos humanos, la jurisprudencia de la Corte Interamericana de Derechos Humanos. En ese fallo, además, la Corte Suprema de Justicia de la Nación reconoció la supremacía legal de los tratados por sobre las leyes nacionales. Con posterioridad, la reforma constitucional de 1994 reconoció la importancia del sistema internacional de protección de los derechos humanos, con la incorporación, en el artículo 75 inc. 22 de la Constitución Nacional, de tratados y declaraciones internacionales de derechos humanos otorgándoles una jerarquía constitucional.

${ }^{9}$ Las "leyes del no" son legislaciones que la lucha socioambiental logró imponer en diferentes provincias, dichas normativas prohíben algún aspecto vinculado a la explotación metalífera a cielo abierto: Chubut (Ley 5001 de 2003); Tucumán (Ley 7879 de 2007); La Pampa (Ley 2349 de 2007); Córdoba (Ley 9526 de 2008); San Luis (Ley 634 de 2008); Tierra del Fuego (Ley 853 de 2012). En el caso de La Rioja (Ley 8137 de 2007) y Río Negro (Ley 3981 de 2005), ambas fueron posteriormente derogadas en 2008 y 2011 respectivamente. ${ }^{10} \mathrm{El}$ análisis que realiza Seoane del tratamiento internacional de lo ambiental se extiende hasta la Conferencia de Naciones Unidas conocida como Río+20 de 2012.

${ }^{11}$ El principio 15 de la Declaración de Río afirma: “Con el fin de proteger el medio ambiente, los Estados deberán aplicar ampliamente el criterio de precaución conforme a sus capacidades. Cuando haya peligro de daño grave o irreversible, la falta de certeza científica absoluta no deberá utilizarse como razón para postergar la adopción de medidas eficaces en función de los costos para impedir la degradación del medio ambiente". En la legislación nacional, este principio fue incluido en la Ley General del Ambiente sancionada en 2002.

${ }^{12}$ Algo que lo diferencia del "principio de prevención", también incluido en la Declaración de Rio. La idea de prevención, por el contrario, opera sobre la certidumbre, se aplica en situaciones ante las cuales los efectos o impacto de la acción son conocidos, pueden valorarse y corregir anticipadamente.

${ }^{13}$ La autora advierte que a pesar del verbo utilizado en la Declaración de Rio ("deber"), la formulación hay que considerarla débil al estar condicionada por las "capacidades" de los Estados y el "costo económico aceptable" 


\section{Referencias bibliográficas}

Alimonda, Héctor (2016) "Brasil, extractivismo y la catástrofe ambiental del río Doce", http://www.vocesenelfenix.com/content/brasil-extractivismo-y-la-cat\%C3\%A1strofeambiental-del-r\%C3\%ADo-doce accesado el 10 de noviembre de 2017.

Altvater, Elmar (2011) Los límites del capitalismo. Buenos Aires: Mar dulce.

Antonelli, Mirta Alejandra (2009) "Minería transnacional y dispositivos de intervención en la cultura. La gestión del paradigma hegemónico de la 'minería responsable y desarrollo sustentable"”. En Maristella Svampa y Mirta Antonelli (editoras) Minería transnacional, narrativas del desarrollo y resistencias sociales. Buenos Aires: Biblos: 51-101.

Antonelli, Mirta Alejandra (2016) "Del pueblo elegido y el maná escondido. La minera en San Juan (Argentina)". Tabula Rasa, 24: 57-77.

Barrientos, María Julia (2012) "El conflicto socio ambiental al calor de las transformaciones del capital. El caso de la Audiencia Pública, Uspallata, Mendoza”. Tesis de la Licenciatura en Sociología, Fac. de Ciencias Políticas y Sociales, UNCuyo, http:// bdigital.uncu.edu.ar/4803 accesado el 6 de octubre de 2017.

Bellotti, Mirta Liliana (2011) "Minería a cielo abierto versus glaciares en alerta roja en Argentina”. Revista de Derecho de Daños, 1:391-437.

Blichner, Lars Chr. y Anders Molander (2008) “Mapping Juridification”. European Law Journal, 14(1):36-54.

Chinigioli, Evangelina, Julia Barrientos y Micaela Fachinetti (2014) "Las Narrativas en torno al 'desarrollo'. La disputa de sentidos por la 7722”. VII Jornadas de Economía Crítica. La Plata.

Drnas de Clément, Zlata, dir. (2008) El principio de precaución ambiental. La práctica argentina. Córdoba: Lerner Editora S.R.L.

Elizalde, Antonio (2015) “Grito de la tierra, grito de los pobres, la propuesta ética de Francisco: una recuperación de los aportes latinoamericanos a la construcción de nuestra Casa Común. Desenvolvimento e Meio Ambiente, (35):121-146.

Esteve Pardo, José (2006a) "La intervención administrativa en situaciones de incertidumbre científica. El principio de precaución en materia ambiental”. José Esteve Pardo (coord.) Derecho del medio ambiente y administración local. Madrid: Fundación Democracia y Gobierno Local: 201-212. 
Esteve Pardo, José (2006b) “El derecho del medio ambiente como derecho de decisión y gestión de riesgos". Revista electrónica del Departamento de Derecho de la Universidad de La Rioja, (4):7-16.

Harvey, David (2004) “El nuevo imperialismo: acumulación por desposesión”. En L. Panitch y C. Leys (comps.) El nuevo desafio imperial. The Socialist Register. Buenos Aires: CLACSO: 99-129.

Hinkelammert, Franz (2006) "La globalidad de la tierra y la estrategia de la globalización”. En Atilio Borón, Javier Amadeo y Sabrina González (comps.) La teoría marxista hoy: problemas y perspectivas. Buenos Aires: CLACSO: 365-378.

Lander, Edgardo (2011) "La economía verde. El lobo se viste de cordero", http:// www.tni.org accesado 15 de septiembre de 2014.

Lavarello, Julieta (2016) Constitucionalidad de la ley 7722 de Mendoza a la luz del principio precautorio ambiental. Tesis de grado de la Carrera de Abogacía. Facultad de Derecho, Universidad Nacional de Cuyo.

Leite Lopes, José Sérgio (2006) "Sobre processos de "ambientalização" dos conflitos e sobre dilemas da participação”. Horizontes antropológicos (25): 31-64.

Machado Aráoz, Horacio (2012) "Los dolores de Nuestra América y la condición neocolonial”. Revista OSAL (32): 51-66.

Martín, Facundo y Lucrecia Wagner (2013) “Agua o minería. Determinaciones y movilizaciones en la construcción pública del conflicto ambiental en Mendoza”. En Gabriela Merlinsky (comp.) Cartografías del conflicto ambiental en Argentina. Buenos Aires: CLACSO/CICCUS: 287-320.

Merlinsky, Gabriela (2009) “La causa 'Beatriz Mendoza' y el conflicto por la apropiación de los recursos naturales en la Región Metropolitana de Buenos Aires: jjusticia para el Riachuelo?”. Ponencia presentada en el XXVII Congreso ALAS Latinoamérica interrogada. Buenos Aires, sin publicar.

Merlinsky, Gabriela (2013) "La espiral del conflicto. Una propuesta metodológica para realizar estudios de caso en el análisis de conflictos ambientales”. En Gabriela Merlinsky (comp.) Cartografías del conflicto ambiental en Argentina. Buenos Aires: CLACSO/ CICCUS: 61-86.

Moreno, Camila (2013) “Las ropas verdes del rey. La economía verde: una nueva fuente de acumulación originaria”. En Miriam Lang, Claudia López y Alejandra Santillana (comp.) Alternativas al capitalismo/colonialismo del siglo XXI. Quito: Fundación Rosa Luxemburg/Abya Yala: 63-97. 
Onofrio, Sergio (2009). "La construcción de la identidad en los movimientos asamblearios de resistencia a la actividad minera metalífera. Caso San Carlos". Ponencia presentada en el XXVII ALAS Latinoamérica interrogada. Buenos Aires, sin publicar.

Onofrio, Sergio et al. (2010) "El poder instituyente de los movimientos sociales. El caso de las asambleas ciudadanas y la Ley 7722 en la Provincia de Mendoza". Presentado en II Jornadas Internacionales de problemas latinoamericanos. Córdoba, sin publicar.

Rodríguez Salas, Aldo (2004) "El principio precautorio y los conflictos ambientales en el derecho ambiental mendocino". En Ana Scoones y Eduardo Sosa (comp.) Conflictos socio-ambientales y políticas públicas en la provincia de Mendoza. Mendoza: Oikos Red Ambiental: 60-78.

Roux, Rhina (2008) "Marx y la cuestión del despojo. Claves teóricas para iluminar un cambio de época". Revista Herramienta (38), http://www.herramienta.com.ar/revista-herramienta-n-38/marx-y-lacuestion-del-despojo-claves-teoricas-para-iluminar-un-cambio-de-e accesado el 13 de agosto de 2009.

Salomone, Mariano Javier (2017) "Un impasse en medio de la tormenta. La defensa del agua en Mendoza al reparo de un fallo judicial". Presentado en las Terceras Jornadas de Sociología de la Facultad de Ciencias Políticas y Sociales, Universidad Nacional de Cuyo, Mendoza. Sin publicar.

Seoane, José (2017) Las (re)configuraciones neoliberales de la cuestión ambiental. Buenos Aires: Ediciones Luxumburgo/GEAL/IEALC.

Seoane, José, Emilio Taddei y Clara Algranati (2013) Extractivismo, despojo y crisis climática. Buenos Aires: El Colectivo/Herramienta/GEAL.

Suprema Corte de Justicia de Mendoza (16/12/2015) "Minera del Oeste S.R.L. y ot. c/ Gbno. de la Provincia p/ acción inconstitucionalidad”, http://www2.jus.mendoza. gov.ar/listas/proveidos/vertexto.php?ide=4519771086 accesado el 20 de marzo de 2016 .

Svampa, Maristella y Mirta Antonelli (2009) Minería transnacional, narrativas del desarrollo y resistencias sociales. Buenos Aires: Editorial Biblos.

Svampa, Maristella y Enrique Viale (2014) Maldesarrollo. Buenos Aires: Katz.

Voces de alerta (2011) 15 Mitos y realidades de la minería transnacional en Argentina. Buenos Aires: Editorial El Colectivo/Ediciones Herramienta.

Wagner, Lucrecia (2014) Conflictos socioambientales. La megaminería en Mendoza 18842011. Buenos Aires: Universidad Nacional de Quilmes.

Williams, Raymond (2000) Marxismo y literatura. Barcelona: Península. 


\section{Fuentes periodísticas}

El Sol (11/10/2015) “El pedido contra la Ley 7.722 ya pasó por tres jueces”, http:// www.elsol.com.ar/nota/247043 accesado el 22 de marzo de 2016.

El Sol (17/12/2015) “Es un fallo huérfano de argumentos jurídicos", http://www. elsol.com.ar/nota/252461 accesado el 26 de marzo de 2016.

El Sol (25/11/2015) "El impacto del mayor desastre ambiental de Brasil ya llegó hasta el mar", http://www.elsol.com.ar/nota/247082 accesado el 22 de marzo de 2016.

Los Andes (16/12/2015a) "La Corte provincial decretó la constitucionalidad de la ley antiminera 7722", http://www.losandes.com.ar/article/triunfo-ambientalista-la-corte-provincialdecreto-la-constitucionalidad-de-la-ley-antiminera-7722, accesado el 25 de febrero de 2016.

Los Andes (16/12/2015b) "Mendocinos festejaron en la peatonal Sarmiento el fallo de la Corte", http://www.losandes.com.ar/article/mendocinos-festejaron-en-el-kilometro-cero-el-fallo-de-la-corte accesado el 3 de abril de 2016.

Los Andes (6/11/2015) “Tragedia en Brasil por un derrame minero: 17 muertos y decenas de sepultados bajo un 'lodo tóxico"', http://www.losandes.com.ar/article/tragedia-en-brasil-por-un-derrame-minero-17-muertos-y-decenas-de-sepultados-bajoun-lodo-toxico accesado el 15 de abril de 2016.

Mdz (17/12/2015) “La Cámara de Servicios Mineros calificó a la decisión de la Suprema Corte como un 'grave atentado a la democracia", http://www.mdzol.com/nota/646698mineros-calificaron-de-politico-el-fallo-a-favor-de-la-7722/ accesado el 2 de mayo de 2016.

Unidiversidad (17/12/2015) "El fallo por la 7722 sería de referencia para el país", http://www.unidiversidad.com.ar/el-fallo-por-la-7722-seria-de-referencia-para-elpais accesado el 15 de mayo de 2016.

\section{Cómo citar este artículo:}

Salomone, Mariano Javier (2019): “Todo fallo es político. Fragmentos de ecología política en la Suprema Corte de Justicia de Mendoza”. Revista Perspectivas de Políticas Públicas vol. 8 No 16:365-393 